\title{
Redefining Evolution: Life Beyond the Limits of Neo-Darwinian Theory
}

\author{
Jay Elliott Silverstein \\ Anthropologist, School of Advanced Studies, University of \\ Tyumen, Russia
}

\begin{abstract}
Neo-Darwinian evolutionary theory is fixed in scientific thought as a dogma despite the lack of congruence with some observable and theoretical phenomena related to culture, epigenetics, abiogenesis, and agenetic life. Even the most current versions of evolutionary theory fall short in explaining a range of scenarios that lay outside Neo-Darwinian principles. Proposed here is a recontextualization of Darwinian theory within a new paradigm that focuses not on the biomechanics of evolution but on the existence of various mediums for transmitting Coded Information Networks through time and space. Following this revised perspective, evolution is not a uniform process but rather one defined by a series of overlapping stacked systems for carrying information organized with stepwise increased complexity and corresponding increased potential for manipulating and moulding matter into more complex forms. Panevolutionary Theory identifies three different types of evolution that, while containing different modes of operation, describe the processes used for creating and maintaining life in all its various forms. Phusitic Evolution describes the emergence of life through the dynamics of inorganic compounds, Zoetic Evolution models the propagation of life through molecular biological processes, and Noetic Evolution explains organisms and designed intelligent systems in which the knowledge itself directs the processes required for existence. This Panevolutionary perspective allows observable and theoretical phenomena related to 'Big History' and the complexity of life, including human behaviour, to be explained under unifying principles while resolving paradoxes and inconsistencies in the current attempts to apply the Neo-Darwinian paradigm as universal law.
\end{abstract}

Social Evolution \& History, Vol. 20 No. 2, September 2021 3-26

(C) 2021 'Uchitel' Publishing House

DOI: $10.30884 / \mathrm{seh} / 2021.02 .01$ 
Keywords: evolution, panevolution, informatics, Darwinism, neoDarwinism, information networks.

\section{THE CHORUS OF DISCONTENT}

The Theory of Evolution explains the variety of life on earth using a handful of principles rooted in Darwin's original thesis. Since Darwin, adjustments to the concepts of Darwinian Evolution have accommodated scientific, mathematical, and philosophical advances resulting in versioning updates such as Neo-Darwinism, Neoevolution, Sociobiology, the Modern Synthesis, the Extended Synthesis, and Inclusive Evolutionary Synthesis (Danchin et al. 2019). Still, one of the fundamental difficulties in evolutionary studies lies in reconciling human culture with the natural selection of genes and thus integrating the biosphere (Vernadsky 2012) within the sphere of 'Big History' (HughesWarrington 2005; Nazaretyan 2005; Snooks 2005b, 2005a). More recent and related conflicts have arisen with the integration of the growing body of research on epigenetic and non-genetic inherited attributes into the broader theory of evolution (Carey 2012; Laland et al. 2014).

In most cases, tweaks and adjustments to the Theory of Evolution strive to fit these revelations within Darwin's box because of a dogmatic embrace of the universality and primacy of the Darwinian paradigm. However, many scholars point out that some assumptions in the Modern Synthesis of Neo-Darwinism are not consistent with what we now understand and observe about the processes of change in lifeforms (Balon 2004; Cobb 2007; Danchin et al. 2011; Enriquez and Gullans 2016; Huneman 2019; Mazur 2015, 2019; Noble 2006; Pigliucci et al. 2010; Pookottil 2013; Ryan 2002; Shapiro 2013; Snooks 2003; The Third Way 2020). There are phenomena known now that do not in any significant way coincide with the NeoDarwinian paradigm. I propose here that science recognizes the limitations of the current paradigm and that the Darwinian theory derivatives are more appropriately considered a subcategory of a more extensive universal Panevolutionary process (see Sahlins and Service 1960; Carneiro 2005) in which information is managed and propagated through time through a variety of mediums, genes being but one of the options that exist among numerous other observable and theoretical mediums. Likewise, Natural Selection is but one means of many ways of propagating information, and that selection need not be based in any pragmatic way on fitness to a given natural environment. The consequence of this paradigm shift is somewhat counterintuitive since it switches the emphasis from the organism or even the DNA to the information carried by whatever vehicle for accomplishing that task is 
in operation, whether by what Bernard Stiegler (2018b) might refer to as noetic and non-noetic intelligence or by unconscious selforganizing natural phenomena (Snooks 2007).

There is a growing chorus of discontent among scientists from various disciplines regarding the universality of the Theory of Evolution. Since Darwin's initial thesis, the theory has undergone its own adaptive process evolving to accommodate the flood of discovery from biology, genetics, ecology, zoology, paleontology, anthropology, and sociology ( $c f$., Harris and Laade 1968; Mayr and Provine 1998; Sanderson 2007). There are, however, fundamental parameters in the Neo-Darwinian model that cannot be crossed, and to do so would undermine the model's defining character. Foremost of these evolutionary components is the relationship between processes of Selection and Genetic Inheritance. Suppose the Modern Synthesis has little or tangential relevance to even one observed or theorized process in the organization and propagation of life on earth. In that case, NeoDarwinism is not a universal law but rather a subset of some other overarching process. Darwinian evolution, therefore, could be viewed as pertaining only to a particular set of circumstances rather than all circumstances of life, real or realistically imaginable.

Panevolutionary Theory suggests different types of evolution that, while containing different modes of operation, describe processes capable of creating and maintaining life in its potential forms. Phusitic Evolution describes the emergence of life through the dynamics of inorganic compounds, Zoetic Evolution models the propagation of life through genetic and bio-epigenetic processes, and Noetic Evolution explains organisms and designed intelligent systems in which the knowledge itself directs the processes required for existence.

The Neo-Darwinian paradigm that dominates scientific thought is, without a doubt, an extremely robust model for explaining speciation following the biomechanics of genetics and the relationship between phenotype and environment. However, Homo sapiens consistently run afoul of Darwinian evolution because of our ability to manipulate environments, manipulate phenotypic expression, and manipulate genotypes with evermore efficiency and deliberate intent. Even worse, as individuals and groups, we are prone to making decisions that defy evolutionary precepts of survival and propagation. Darwin himself struggled with this paradox (Darwin 1859: 201; 1889: chap. IV) and, despite the advent of the field of Sociobiology (Wilson 2004), the construction of a parallel meme-based cultural evolution (Dawkins 1989), and the development of epigenetic inheritance theory (Carey 2012), we have not resolved the fundamental paradoxes of humanity 
and the Theory of Evolution. Ultimately, human survival must be attributed to the inheritance of favorable traits and behaviours, and ultimately this is tied to the genetic foundation of organic existence. However, the logic that humanity must in our modern condition conform to Darwinian parameters forms a Cartesian Circle in which, Evolution created us; we are able to ascertain that we exist; therefore, our sentience was selected for by Darwinian evolution; therefore, Darwinian evolution exists because we exist to conceive of it; and therefore, because evolution accounts for our origin, all past and future evolution and behavior must be explained under Darwinian laws. The logical fallacy is, of course, an assumption of universality and uniformity of the Darwinian forces. Thomas Huxley compared Darwin's hypothesis to the hypotheses on orbits by Ptolemy, Copernicus, Kepler, and Newton: 'What if the orbit of Darwinism should be a little too circular? What if species should offer residual phænomena [sic], here and there, not explicable by natural selection? Twenty years hence naturalists may be in a position to say whether this is, or is not, the case' (Huxley 1860: 569). Huxley was prescient in this analysis.

There is no logical rationale why natural selection or genetics must be necessary to all aspects of evolution - as David Hume (2007) points out, humanity is prone to working on assumptions of continuity that are matters of habit. Academe likes laws that cover all contingencies, and it is reticent to demote accepted laws to a secondary status that might relate only to a specific time and set of circumstances. If, however, observations and logical projections of the future demonstrate that life can exist and propagate outside of the Modern Synthesis of the Darwinian model, we must consider that there is another way to model evolution that has greater universality and that the current paradigm is but a subspecies of a larger set of laws describing the pattern of organic existence and the propagation of living essences through time and space.

\section{PARAMETERS}

Biological Evolution has come to mean 'changes in the heritable traits of a population of organisms as successive generations replace one another' (National Academy of Sciences 2020). A broader definition of evolution not explicitly tied to molecular biology is 'the process by which the frequencies of variants (be they genetic or not) change over generations' (Danchin et al. 2019). Darwinism states that all species of organisms arise and develop through the natural selection of incremental, inherited variations that increase the individual's ability to compete, survive, and reproduce. Neo-Darwinism represents the subsequent de- 
velopments associated with the integration of Mendelian genetics. The Modern Synthesis merges Darwinian concepts of selection with genetics. In the 1940s and 1950s, JBS Haldane, Ronald Fisher, Sewall Wright, Ernst Mayr, Theodosius Dobzhansky, George Simpson, Julian Huxley, Bernhard Rensch, and others further elaborated the theory by paying greater attention to population genetics (Cain 2009; Danchin et al. 2019; Gayon 1998; Mayr 1998; Huneman 2019).

Culture can be defined as 'the system of socially transmitted patterns of behaviour, preferences, and products of animal activities that characterize a group of social animals.' Cultural Evolution is then the 'change, through time, in the nature and frequency of these socially transmitted preferences, patterns, or products of behaviour in a population' (Jablonka et al. 2014; Richerson and Boyd 2005). Danchin and his colleagues (Danchin et al. 2019) have proposed,

...[an] extension of the Modern Synthesis that includes all components of inheritance and their interactions. Its ambition is to incorporate any known forms of parent-offspring resemblance, including epigenetic, ecological and cultural inheritance, parental effects of all sorts, as well as the inheritance of microbiota or the effects of any molecular memory system such as, for instance, in prions.

This effort expands the understanding of evolution by confronting what they term Genocentrism. Yet, it does not fundamentally change core principles of the Darwinian model. Instead, it stretches open the reach of Darwin's umbrella to reconcile the Theory of Evolution with non-genetic autopoietic existence. There are, however, phenomena that fall too far from Darwinian precepts to be usefully explained even with the most current adjustments to evolutionary theory.

\section{ABIOGENESIS (PHUSITIC EVOLUTION)}

The Modern Synthesis strongly emphasizes the controlling role of genetics in evolution yet, we know with a great deal of theoretical certainty backed by chemical experimentation (Canavelli et al. 2019; de Jong 1932; Snooks 2005b) that life formed from non-organic chemicals. Within the matrix of the primordial world were the materials and the forces to direct the formation of increasingly more complex organic compounds leading to the abiogenetic creation of DNA and consequently autopoietic life (Barbieri 2003; England 2013). This evolutionary step represents a horizon that, when crossed, expanded the realm of possibilities of what could and what would exist in the universe. The process of abiogenesis could be categorized as a form of 
selective environmental pressure leading to the formation of these chemicals; however, it was an agenetic process perhaps no more evolutionary in a Darwinian sense than crystal formation. Thus, the original evolution of life falls entirely outside the parameters of a NeoDarwinian model, thereby demonstrating that life can be achieved from a non-organic origin through the increasing organizational complexity of molecules under the physical laws that guide inorganic chemistry - that is, Phusitic Evolution.

\section{CULTURE (NOETIC EVOLUTION)}

Culture is another sphere of evolution that fits uneasily in the Darwinian paradigm. There are a few options for explaining culture in NeoDarwinian terms (Andersson 2011; Mazur 2015; Cobb 2007; Richerson and Boyd 2005). Culture can be explained as the phenotypic expression of the underlying genome, as supported by Sociobiology. Culture can be viewed as an epigenetic means of inheritance as described in the Modern Synthesis. Or, culture can be modeled as a parallel means of evolution connected to genetic evolution, but also apart from it as explained in the Inclusive Evolutionary Synthesis or memetics. In all cases, culture is seen as a force operating under Darwinian principles of variation, inheritance, and selection. I would argue that, while aspects of culture could fall into any one of these classifications that, as a whole and given that culture can be discontinuous, abstract, symbolic, cumulative, and transferable with no biological hereditary or physical relation, culture is a creature alien to many of the precepts of evolution. Even accepting intentionality as a selective environmental force at all scales of species and interspecies interaction (genetic, individual, and population), it is difficult to corral the reality of a highly arbitrary and often intentional phenomenon like culture within the confines of Darwinian principles of natural selection, gradualism, and generational inheritance (Darwin 1859: 201; Enriquez and Gullans 2016; Wilson et al. 2014).

Since collective ideas determine culture, the concept of culture must include the cumulative knowledge of humankind presently reified on the World Wide Web. The proliferation and accessibility of knowledge mean that this information is actionable in any place or time where there are the appropriate mechanisms to use that knowledge. Consequently, knowledge can direct the reorganization of energy and matter based on human intent (e.g., Enriquez and Gullans 2016; Woolfson 2000). Information is routinely used to manipulate the genetic and biological organization of species, and this is now a normal part of culture (e.g., Garcia and Trinh 2019). The code for an organ- 
ism could theoretically be transmitted through time and space to be reconstructed and, if desired, modified elsewhere, removing the constraints of time and space in the propagation of life and replacing it with human intentionality. The control of knowledge, therefore, has usurped the genome-environmental selection mechanism of evolution. Mechanisms outside of those that defined evolution in the past promise a future far stranger than any Darwin and his contemporaries could have imagined - this is now a cultural reality. Genetic engineering is far outpacing the scientific adherence to a Victorian mode of thought. In this new paradigm, it is taken for granted that genetic codes are recorded in electronic memory, thereby preserving the code in a new medium for use at human discretion. Much of the theorizing and discourse related to these biotechnological breakthroughs focus on explaining this explosion of knowledge within a Darwinian framework or suggesting that laboratory work is an exception. Ironically, Darwin's theory disentangled human existence from divine conscious design, alleviating humanity from the necessity of theological genesis (Dawkins 2015; Mayr 1991); yet, we now find humanity fully engaged in assuming the God-role by mastering capabilities that allow the conscious construction of the structures of life (Enriquez and Gullans 2016; Stiegler 2018a).

The prospects for the future of life become more challenging when we consider the rapid pace of development of Artificial Intelligence and Machine Learning (Penrose 1999; Woolfson 2000). While we are not close yet, recent advances in synthetic memory management provide a reasonable theoretical base for consideration of synthetic and biosynthetic neural systems capable of matching and exceeding the human brain's capacity (Makin et al. 2020; Rai et al. 2020; Tizno et al. 2019). If we accept that this Science Fiction-like prospect is at least theoretically possible if not incipient, we must consider the prospect of human intelligence existing outside the biological body and accept that this prospect must somehow fit within the context of an evolutionary paradigm. The Dualism of mind and body that dominated much of philosophy and theology before Darwin must now be reconsidered in terms of extrasomatic existence (Kaku 2014). Autonomous non-corporeal life challenges the definition of life and many of the precepts of the various syntheses of evolutionary theory.

Maintaining the Darwinian paradigm would require a broadened definition of environment that includes arbitrary circumstances such as engineered electronic network systems or mediums of wave transmission. It would also require expanding the concept of selection to include every arbitrary whim that results in some new variant of an 
organism. Such definitions push these controlling variables beyond any parameters of Darwinian-based theory. Applying the above 'exceptions' to Neo-Darwinian principles engages the current paradigm in that logical circle again where the fact that an organism exists can only be due to selection and that selection is proved by the organism's existence. The cases described above in which Neo-Darwinian theory fails to provide a causal description of reality can be addressed if evolutionary theory shifts from the physiological structure of life forms to their organizing principle (i.e., the coded information underlying the form). It has become apparent in the twenty-first century that these codes can be carried in other than biogenetic systems and still retain their viability for defining a living organism and directing its reproduction by means external to the natural reproductive mechanisms of the species. If the information carried by the organism is the measure of evolution, the mechanisms for carrying and translating the informational infrastructure of an organism can be viewed as an epiphenomenon. In other words, it is not the briefcase that is important but the documents that lie within it. Rather than being constrained by the limitations of a genocentric perspective, Panevolutionary Theory encompasses agenetic Phusitic Evolution, Neo-Darwinian Zoetic Evolution, and cognitive Noetic Evolution. It offers a cohesive model of evolution parsimonious with the various ways matter and energy are used to construct life, even those reasonably projected into the future.

\section{EVOLUTION IN THE TWENTIETH CENTURY}

The first half of the twentieth century stands out for the scientific revelations that fundamentally changed humanity's relationship to the earth and universe. The ideas planted in the nineteenth century blossomed into physical realities in the century that followed. Every field of scientific inquiry led to the inexorable acceptance of Homo sapiens being subject to the same laws of physics and natural reality as any other matter. Science forced open the door that had been set ajar by Darwin, unhinging humanity from the tenacious reliance on supernatural explanations for our existence (Agar 2012; Mayr 1991). From this unification of diverse fields came a heightened awareness of the holistic nature of astronomical, geological, biological, and sociological history, which fostered new theoretical perspectives designed to capture the contiguity of existence. Consonant with this revolution was the recognition of a biosphere that is fully synergetic with the atmosphere and exosphere. As a constituent of the biosphere, humanity could be defined by our place in the cycles and systems of energy and matter. Yet, there was also 
recognition that humanity played a unique role in the earth's dynamic systems (Vernadsky 1944). Vernadsky (2012: 22) elaborated on the anthropogenic dynamics noting, 'Through his labour and his consciousness, the biosphere is in a process of transition to the noosphere. Human is creating new biogeochemical processes, which never existed before.' In recognition of the degree of the impact human activity had on the planet, some scholars nominally, and now with increasing acceptance, identify the period of earth history commencing with the Industrial Revolution as the Anthropocene Epoch (Teilhard de Chardin 1955; Steffen et al. 2011).

Newtonian laws of physics constrain non-sentient matter within fixed natural systems like the Hydrologic Cycle or Biogeochemical Cycle. Before the twentieth century, most attempts to model the evolution of human societies used vector-like unilineal socio-technological trajectories, not unlike natural cycles (e.g., Morgan 1877; Spencer 1990; Tylor 2010). These concepts were rejected by the Boasian School of anthropology, which recognized the uniqueness of cultures (Adams 2016). A schism arose in the young field of anthropology between schools that viewed societies as evolving along the lines of Historical Particularism and those favoring a more empirical Cultural Materialist point of view. Throughout the twentieth century, no theoretical perspective was more impacted by this schism than that of evolution.

Neoevolutionist perspectives recognized that ecological cycles could be reduced to an analysis of energetic systems at a fundamental level. Following this, Natural Selection could be interpreted as the success of a species predicated on its ability to optimize energy within its ecological niche (White 1943). Leslie White (1959: 12-13) defined culture as an extrasomatic means of adaptation. Neoevolutionary thought aligned with the Positivist ideas by emphasizing cultural energetics as expressed in White's Law of Cultural Development: 'culture advances as the amount of energy harnessed per capita per year increases, or as the efficiency or economy of the means of controlling energy is increased, or both' (White 1959: 56). White expressed his law in the 'simple formula: $\mathrm{E} \times \mathrm{T}=\mathrm{P}$, in which $\mathrm{E}$ represents energy expended per capita per unit of time, $\mathrm{T}$ the technological expenditure, and $\mathrm{P}$ the magnitude of the product per unit of time' (White 1943: 337). Cultural Ecology unified energetics directly with ecological adaptation to explain variations in adaptive strategies consistent with a multilineal perspective (Sanders 1962; Steward 2008; White 1959). A variant school of Cultural Materialism encapsulated Neoevolutionary ideas 
under the broader concept of economy (Harris and Laade 1968) and World Systems Theory (Sanderson 1999), elucidating the processual aspects of culture change rooted in Neo-Marxian Theory.

Contemporaneous with the theoretical effort to explain the material dynamic of humanity and environment were the genetic revelations surrounding the decoding of DNA. Sociobiology followed suit as a subdiscipline of anthropology aimed at elucidating the logical bridge between genetics and behavioural studies through rigorous statistical rationales explaining behaviour as a genetic imperative (MacArthur and Wilson 2001; Wilson 2000). Dawkins (1989) epitomized this genocentric perspective with his Selfish Gene aphorism. Yet, even the most ardent genocentrists recognized the exceptional position of culture. To address this, Dawkins (1989: 196) introduced a second aphorism, the meme, that, in essence, proposed separate but related forces of evolution.

In the waning years of the twentieth century, Wilson (1998a, 1998b) evoked the intellectual spirit of the Enlightenment by reviving Holton's (2000: 160-69) idea of the 'Ionian Enchantment' and Whewell's (1840) discourse on scientific language to resurrect 'consilience' as the centerpiece of his quest for universality. It was an attempt to reconcile memes and genes in the epistemology of evolution. Wilson concluded (1998a: 292-93), "the human condition is the most important frontier of the natural sciences. Conversely, the material world exposed by the natural sciences is the most important frontier of the social sciences and humanities. The consilience argument can be distilled as follows: The two frontiers are the same.' Yet, it seemed after 100 years of discovery and theorizing; our theories had come no closer to recognizing a common principle that could unify cultural and genetic evolution. It was not possible under the uniformitarian precepts of Neo-Darwinian Theory.

The twentieth century represents the most dynamic century of scientific discovery thus far in history; however, in many ways, the theoretical and philosophical constructs for these revelations failed to keep pace. It is easier to explain the empirical science behind the creation of a hydrogen bomb than to explain the social science of why our cultures would choose to build it. In the end, it seems that Vernadsky had best summarized the underlying force of culture - it was neither the energy harnessed nor the genetic potential, but rather the enabling corpus of knowledge that guided human will:

The study of all phenomena has a unity, leading to the production of a body of systematized knowledge, the corpus scien- 
tiarum, which tends to grow like a snowball; this corpus includes all systematized knowledge, and is contrasted to the results of philosophy, religion, and art where truth may be revealed intuitively; the systematized history of these activities belongs to the corpus (Vernadsky 1945: 2).

\section{COGNITIVE REVOLUTION}

The evolution of cognitive intelligence offered advantages in plasticity, allowing Homo sapiens to adapt and anticipate environmental stresses through communal action and collective symbolized memory. A consequence of the brain's adaptability was an ability to realign human thought and behaviour in ways that were potentially contradictory to Neo-Darwinian precepts for perpetuating specific genetic attributes. Humans carry neural-hormonal holdovers and homologies of our Darwinian past, like vestigial structural problems associated with upright two-legged tailless posture or convoluted epiglottal reflexes. Elements from our evolutionary history carried in our limbic system can manifest as psychological duress and cultural maladaptation to contemporary society; however, while not free of our genetic antecedents, neither is humanity bound by them. Culture has superimposed norms of cooperative behaviour. We develop drugs, skills, and philosophies that temper, redirect, or suppress certain instinctual behaviours. Through science, humanity devises capabilities to manipulate genetics, transform the earth's environment, share a Cloud consciousness, and create artificial intelligence, thereby taking significant conscious control of selection, reproduction, and environmental stress. Humanity has entered upon a path whereby the circumstances of our origin and the descent of humanity are less and less relevant to our current experience of evolution. The forces that directed the genesis of our species had superimposed upon them a system of intentionality borne of human cognitive intellect that differs not only in magnitude but in essential character from those forces that guided our creation.

Computers offer an apropos analogy for the impact of cognitive awareness on the development of human life. A computer designed to calculate taxes can be reprogrammed with new software to play 3D games. Although now operating in a fashion wholly different from the circumstances of its genesis, the plasticity of the system allowed the same hardware configuration to be put to a completely different and unintended function, a function that might be utterly divorced from the original purpose (Jablonka et al. 2014). In the same way, the human mind, particularly our collective mind in the form of culture, has been reprogramming itself since its creation and most intensively since the Neolithic. Culture drifts ever farther and more rapidly from the condi- 
tions and constraints that existed when it first coalesced. The rules of the evolution game changed and are changing at an ever more rapid pace. While not (yet) lost, we are shedding the concepts of (a) random mutation, (b) the correlation between genotype, phenotype, environment, and survival, and (c) competition being the means of success of an individual or group.

If the mechanisms and processes of cultural and genetic evolution differ as discussed here, then we are forced to accept that there is no common denominator. However, if we believe that a unified theory of evolution offers uses for modelling our future, then it is worth exploring and reformulating a more applicable heuristic. Is there a universal principle that guides the organization, perpetuation, and mutability of life as it applies to humanity? I suggest that the common denominator of Neo-Darwinian Evolution and Cultural Evolution is that both systems function to transmit networks of coded information through space and time ( $c f$. Bonduriansky and Day 2018). Neo-Darwinian evolution uses genetic codes expressed through epigenetic processes and relationships formed by the gradual negentropic organization of chemicals. In contrast, culture uses a diverse and dynamic array of symbolic codes purposefully designed to carry and transmit information such that it can be expressed through mediums that enable other humans (and machines) to ingest and use it. Essentially, both Biological and Cultural Evolution are processes that transmit and use coded information - the rest is circumstantial.

The circumstances of both evolutions, however, are the devil in the details. Information is carried through space-time in coded forms in which each element, be it a codon, a radio wave, an alphabet, or a word, is translated through supporting mechanisms to allow that information to guide the organization and conduct of energy and matter. The emergent complexity of the information carried in the codes is much greater than the literal translation of the individual elements of the code. Since the code is transcribed and acted upon through related non-coded forces (e.g., epigenetic and interpretive), the interpretation and expression of that coded meaning is much greater than the individual symbols. The potential impact on reality of the code is far greater than can be intuited from any single element of the code due to the multiplicative effect of the network in which it resides. An analogy drawn from chemistry would be a molecule that has a specific set of properties (essentially coded information residing with discrete atomic particles), but the state and activity of that molecule will vary according to the non-coded effects of environmental factors such as pressure, temperature, and proximity to other chemicals. The complexity of the code lies not as much in the actual code, which can easily be quanti- 
fied, defined, and structured according to specific 'syntax,' but rather in understanding the expression of the codes as applied to reality. One of the primary efforts of the soft sciences is the use of the fields of humanities and social science to understand the interstitial complexities that translate and express the laws of the hard sciences (e.g., biology, chemistry, and physics) and thereby explore not the mechanics of existence, but the consequences of it. Logically, the greater the complexity of the coded information and the greater the complexity of the external forces associated with its translation, the exponentially greater the possibilities for the complexity of expression. Therefore, complex coded systems have an exponential growth pattern based on the size of the code and the means by which it is transcribed and translated into life and behaviour.

\section{A NEW PARADIGM (PANEVOLUTIONARY THEORY)}

Contrary to the assumptions of Neo-Darwinian Uniformitarianism, the complexity of information management in the Panevolutionary model has horizons in which rules that direct the mechanics of how information is maintained, transmitted, and acted upon radically change. Innovations occur that represent revolutions in the organization and management of matter that achieve emergent characteristics that fundamentally change the nature of what can exist. Marcello Barbieri's (2003) study of organic codes and biosemiotics uses a template to understand the initial pattern of life, observing that the code must have resided within the primordial soup kitchen, coded through natural convention rather than within a genetic code of an existing organism. In the beginning, life did not beget life. The abiogenesis of DNA and autopoietic life was a horizon that, when crossed, expanded the realm of what could and would exist in the universe (Canavelli et al. 2019; Heylighen 2010). Thus, the original evolution of life lies outside the Neo-Darwinian model as an example of Phusitic Evolution. Likewise, modern Biotechnology and Artificial Intelligence offer more than a theoretical reality of intelligent life being built and programmed in designed biological or synthetic organisms. Deliberate construction and the potential for purposefully designed agenetic life should likewise be encompassed within a comprehensive model of evolution (Woolfson 2000). Between abiogenesis and AI lies the advent of one of the most profound horizons of evolution, human cognition.

Culture consists of reinforcing positive feedback loops where the interplay between the cognitive capabilities of humanity and the information that humanity accumulates through a wide variety of systems of coding and transmission (e.g., language, writing, art, poetry, etc.) build ad infinitum. The application of this information allows 
purposeful agency in the management of the phenotype-environment relationship. Thus, humanity consciously began reprogramming itself to live according to a worldview of its own creation. This is precisely what Cobb (2007: 43) points out as an issue in evolutionary studies when he concludes that "we do not actually believe that our scientific interpretations of the data are simply the results of the environment selecting among genetic mutations.' The formation of culture has indeed been greatly influenced by its formative precursors and the Darwinian forces that oversaw its genesis, but increasingly culture is influenced by metaphysical and existential ideas, scientific comprehension, creativity, technological innovations, imagination, and delusion that adapt the environment to our desires rather than depending on our biogenetic make-up to adapt to the environment. This is done with an increasing emphasis on propagating knowledge by other than corporeal means. The ability to symbolize and abstract cognitive experience represents one of the fundamental points of transition between evolutionary horizons - from this point forward, information that could determine the fate of a species could be created, modified, transferred, and acted upon in an agenetic medium operating under non-NeoDarwinian principles.

Realigning our emphasis from the means in which information is transmitted to the importance of the information itself makes a difference in how we approach scientific thought and how we accept the wisdom coded and transmitted through conduits of culture such as art, stories, science, religion, crafts, music, economics, etc. From this paradigm, there is no inconsistency between genetic and cultural evolution; they are the same as Wilson (1999) noted in his search for consilience. Both aspects of evolution shape matter and energy into coherent forms perpetuated through time, the only difference being that they use different conduits following different rules to make that reality. One could make an argument that a human being and a poem are both an organization of energy and matter that have no particular significance to the universe at their moment of existence and creation; however, whatever aspect that might endure beyond that moment, as suggested in memetics, is the measure of 'evolutionary success.' It needs have no contiguity in time and space - that poem may have been written in cuneiform 3000 years ago and then be rediscovered and given meaning by a researcher or author from the other side of the planet. Culture is essentially the ordering of matter and energy in the form of symbols that can be acted upon; it is just a highly structured and complex corpus that carries meanings and abilities beyond what could evolve under the laws of nature and natural convention. 
Under a Coded Information Networks (CIN) rubric, Panevolution can be broken down into epochs based on information management systems rather than periods defined by geology, the environment, or the character of life on earth. Four coarse phases that capture key transformations in how information is carried and transmitted are proposed as epochs of a Panevolutionary universal model. A CIN defines a mechanism that perpetuates existence on earth by layering higher orders of information management systems based on their complexity (Fig. 1).

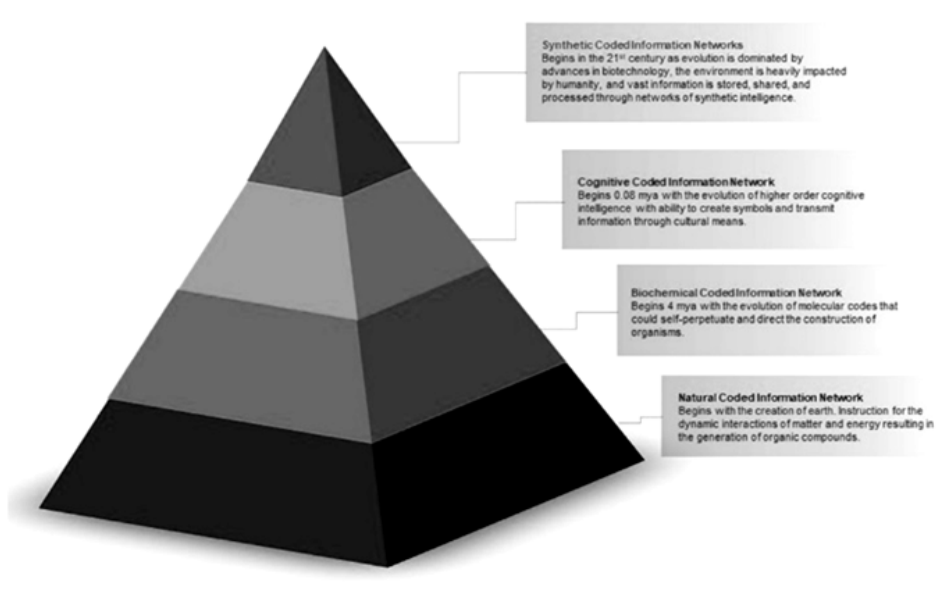

Fig. 1. The complexity hierarchy of Coded Information Networks (CIN)

I. Natural CIN (N-CIN): This represents foundational forces capable of creating information systems through abiogenesis that I have named Phusitic Evolution. This is consonant with Natural Convention (Barbieri 2003) and Wilson's (2005) discussion of the lowest orders of Biological Organization.

II. Biomolecular CIN (B-CIN): This CIN marks the introduction of Zoetic Evolutionary phenomena with the advent of the coding of information as complex organic compounds and the arrival of cellular metabolism to replicate, mutate, and actualize the codes. This epoch aligns with Neo-Darwinian theory and encompasses a range of epigenetic means of inheritance. This matches the second order of organization in Wilson's (2005) discussion on Biological Organization.

III. Cognitive CIN (C-CIN): This Noetic Evolutionary Epoch is defined by the development of cognitive thought following the advent of humanity, a product of lower orders of evolution that acquired the ability to be self-aware, to symbolize and recode knowledge, to apply creativity to existence, and to transmit this knowledge through extra- 
somatic means. No matter how vast the potential library, kinetic information is limited by the capacity of organic neurological networks augmented with analogue data libraries and the capacity to learn, retain, and apply knowledge. The management of information is limited by the inherent bio-structural organizational and intercommunication limitations of individuals and communities. These structural limitations buffer the ability to ingest and use knowledge because of the individualistic nature of our minds and our relatively short life spans. In other words, there is more knowledge than is digestible by any individual.

IV. Synthetic CIN (S-CIN): This Noetic Evolutionary Epoch is defined by the creation of systems capable of storing and processing vast archives of digital information that either significantly augments the organic cognitive capabilities of our species, or that can act autonomously on information accessible within the network. S-CIN will likely culminate with the advent of non-organic synthetic forms of independent cognitive beings capable of the fundamental processes of reproduction and the capacity for selective self-improvement (e.g., Machine Learning) (cf. Brawer et al. 2017).

\section{THE TREND TOWARD INCREASED POTENTIAL}

The various systems of coding and transmitting information that has evolved on earth have followed a developmental trajectory of increased organization of information and increased potential to use that information to modify the world. The prevailing theory of abiogenesis is that the initial organic compounds that formed the building blocks of life in the prebiotic world became organized into monomers that eventually yielded a DNA-based self-replicating system with a mechanism for adaptation and increasing complexity. Thus, a handful of chemicals drawn from the 94 naturally occurring elements, mostly $\mathrm{C}, \mathrm{N}, \mathrm{H}$, and $\mathrm{O}$, interacting with the primordial environment organized into the building blocks of life. The advent of genetic life introduced a system using four base pairs that could code for 20 amino acids and assemble them into ever more complex proteins. Thus, 64 codons carried the information to construct an estimated $8.0 \times 10^{6}$ different proteins yielding something like $5.0 \times 10^{9}$ different species over the course of the history of life on earth. This suggests a pattern of increasing ability to organize matter into more complex forms and that each subsequent introduction of a system of coded information compounded the potential to manipulate matter and the activities of that matter and energy in the universe.

Human cognition introduced a non-organic means for organizing matter and energy in an open-ended system without theoretical limits 
on scale, complexity, or application of the codes. The human brain alone contains about 86 billion neurons, each carrying perhaps 1,000 potential synapses yielding nearly 100 trillion 'data points' (see Kaku 2014). Computer memory on earth, for instance, holds the potential of something like $6.0 \times 10^{18}$ bytes of memory with much of it connected through the Cloud. Each unit of information stored is related to other bits of information and undergoes a process of interpretation resulting in an exponential level of meaning and use of those symbols far beyond the Boolean binary underpinnings of the code. For practicalities, the Noetic Evolutionary systems currently epitomized by the human brain and its derivative CINs (i.e., C-SIN and S-CIN) have the nearinfinite potential for symbolizing, organizing, and applying data to reality and thus modifying in very real ways the nature of existence. In no way is this meant to imply finalism or a teleological determinism to evolution (see Mayr 1991), but it does indicate a pattern of increasing ability to organize and apply negentropic principles to material existence. If any derived imperative exists in nature or humanity, it is along the path of increased complexity (see Stiegler 2018a).

\section{DISCUSSION}

Charles Darwin (1859: 201) noted, 'if it could be proved that any part of the structure of any one species had been formed for the exclusive good of another species, it would annihilate my theory, for such could not have been produced through natural selection.' Such actions are commonplace in human culture (e.g., bacterial, plant, or animal life designed to exist to support us), but I would argue that Darwin overstates the concern; it does not annihilate the fundamental principles of Darwinian Theory but merely establishes that there are parameters bounding its applicability. Completing Darwin's theory, classified here as Zoetic Evolution, are Phusitic and Noetic Evolution. Together, these theories provide a framework for a comprehensive theory of evolution. Thus, Panevolution encompasses Darwin's propositions as well as life and cognitive intelligence beyond the boundaries of NeoDarwinian existence.

A unifying theory of physics presupposes that all matter and energy are constructed of the same essential elements, whether it be a string whose properties depend on its resonance within multiple dimensions or some other relative interaction of the building blocks of existence. Whatever the case may be, the universe is constructed from a very limited number of fundamental building blocks. Thus, as observed by Vernadsky (1945: 1), culture and all that we think, create, and imagine manifest as matter and energy and are constructs rendered through the ordering of the substrate of the universe. Evolution 
represents the intense ordering of matter to the point where the matter, in the form of life, can modify and reorder itself with or without intent. Therefore, evolution can be seen as a process in which matter and energy are organized in progressively complex systems. This varies from White's Law or the Kardashev Scale of extra-terrestrial civilizations since those focus on the quantity of energy managed rather than the organizational complexity of that energy (Kardashev 1964; White 1943). Darwinian theory describes one evolutionary circumstance, albeit one that has existed on earth for nearly four billion years and one that yielded cognitive thought. Regardless of how the theory is manipulated, Neo-Darwinism is not adequate as a comprehensive theory of evolution. It cannot accommodate all dimensions of culture and, if we accept the increasing complexity of the organization of matter as a measure of evolution, the physical and chemical circumstances of abiogenesis or the likely advent of biosynthetic or abiosynthetic forms of existence.

Our ability to transform our world, redesign life, explore beyond our planet, create and appreciate aesthetics, and contemplate existential questions reveals a purpose if we choose to impose one. Information is an elusive force in that it does not break easily into measurable components with defined and consistent values. For example, the words here have value only in context to the other words here and the context the reader may already carry or otherwise choose to investigate. To many, I suspect, the words on these pages will have no meaning or value whatsoever. But it is the complexity of these systems that creates the multiplicative value and power of knowledge.

In identifying epochs of evolution, I explicitly acknowledge that phases come into being through horizons that transform the rules that govern the propagation of the organization, agency, and activity of matter - the game changes. The quantum effect of the harnessing of knowledge means that the limits of knowledge extend not only to the ability to modify living forms and geo-ecological systems of our planet but to the manipulation and harnessing of the laws of physics to act in ways they might not be able to otherwise act without the intervention of intelligent direction. Perhaps this is best exemplified by our creating artificial elements such as Einsteinium that, by natural law, cannot exist on earth.

Re-evaluating evolution in terms of information management may not revolutionize the study of past speciation, or palaeoanthropology, or physics, but it can help change the way we approach the social sciences and future speciation by providing a theoretical context for moral responsibility regarding the application of science. Evolution in the C-CIN epoch granted humanity the power, ability, and responsibility 
for ordering knowledge capable of determining the fate of the earth and all life on it. Likewise, C-CIN granted humanity the ability to derive moral oversite and accumulate wisdom. In this model, humanity has no escape from the responsibility of agency for what happens in the world. As far as we know, the universe is indifferent since it presumably functions as a pre-Cognitive N-CIN, but humanity needs not be indifferent. Cognition created imagination, and, in this model, imagination is a defining evolutionary force.

\section{ACKNOWLEDGEMENTS}

Gratitude is extended to Stacey Bagdi for her support, encouragement, and patient review of this theory. Thanks to the SAS faculty and Robert Littman for their consultations over the many years of talking about these concepts. Thanks to Alis Rasmussen, Rhi, Alex, and David for their patience and support at the genesis of this theory 20 years past. A special thanks to my teachers and mentors who indoctrinated me to biological and cultural evolutionary theory and genetics, Bill Sanders, Robert Jurmain, Ken Weis, Henry Harpending, Jeff Kurland, Mark Stoneking, George Milner, Robert Eckhardt, David Webster, James Hatch, Pat Johnson, Pat Draper, James Woods, Dean Snow, and Ken Hirth. A special note of thanks to the editors of Social Evolution and History, whose feedback was essential in deriving the final form of this article.

\section{REFERENCES}

Adams, W. Y. 2016. The Boasians: Founding Fathers and Mothers of American Anthropology. Lanham, Maryland: Hamilton Books, an imprint of Rowman \& Littlefield.

Agar, J. 2012. Science in the Twentieth Century and Beyond. History of Science. Cambridge, UK; Malden, MA: Polity Press.

Andersson, C. 2011. Splitting the Replicator: Generalized Darwinism and the Place of Culture in Nature. Journal of Economic Behavior \& Organization 80: 657-69.

Balon, E. K. 2004. Evolution by Epigenesis: Farewell to Darwinism, Neoand Otherwise. Rivista di Biologia Biology Forum 97: 269-312.

Barbieri, M. 2003. The Organic Codes: An Introduction to Semantic Biology. Cambridge, UK; New York: Cambridge University Press.

Bonduriansky, R., and Day, T. 2018. Extended Heredity: A New Understanding of Inheritance and Evolution. Princeton: Princeton University Press.

Brawer, J., Hill, A., Livingston, K., Aaron, E., Bongard, J., and Long, J. H. 2017. Epigenetic Operators and the Evolution of Physically Embodied 
Robots. Frontiers in Robotics and AI 4. URL: http://journal.frontiersin. org/article/10.3389/frobt.2017.00001/full.

Cain, J. 2009. Rethinking the Synthesis Period in Evolutionary Studies. Journal of the History of Biology 42: 621-48.

Canavelli, P., Islam, S., and Powner, M. W. 2019. Peptide Ligation by Chemoselective Aminonitrile Coupling in Water. Nature 571: 546-49.

Carey, N. 2012. The Epigenetics Revolution: How Modern Biology is Rewriting our Understanding of Genetics, Disease, and Inheritance. New York: Columbia University Press.

Carneiro, R. L. 2005. Stellar Evolution and Social Evolution: A Study in Parallel Processes. Social Evolution \& History 4 (1): 136-59.

Cobb, J. 2007. The Limitations of Neo-Darwinism and Evidence for a Whiteheadian Theory of Evolution. Worldviews: Global Religions, Culture, and Ecology 11: 32-43.

Danchin, É., Charmantier, A., Champagne, F. A., Mesoudi, A., Pujol, B., and Blanchet, S. 2011. Beyond DNA: Integrating Inclusive Inheritance into an Extended Theory of Evolution. Nature Reviews Genetics 12: 475-86.

Danchin, É., Pocheville, A., and Huneman, P. 2019. Early in Life Effects and Heredity: Reconciling Neo-Darwinism with Neo-Lamarckism under the Banner of the Inclusive Evolutionary Synthesis. Philosophical Transactions of the Royal Society B: Biological Sciences 374: 20180113.

Darwin, C. 1859. On the Origin of Species. Or the Preservation of Favoured Races in the Struggle for Life. $1^{\text {st }}$ ed. London; New York: John Murray. URL: https://www.gutenberg.org/files/1228/1228-h/1228-h.htm.

Darwin, C. 1889. The Descent of Man and Selection in Relation to Sex. New York: D. APPLETON \& COMPANY.

Dawkins, R. 1989. The Selfish Gene. New ed. Oxford; New York: Oxford University Press.

Dawkins, R. 2006. The Blind Watchmaker: Why the Evidence of Evolution Reveals a Universe without Design. London: Penguin science.

de Jong, H. G. B. 1932. Die Koazervation und ihre Bedeutung für die Biologie. Protoplasma 15: 110-73.

England, J. L. 2013. Statistical Physics of Self-Replication. The Journal of Chemical Physics 139: 121923.

Enriquez, J., and Gullans, S. 2016. Evolving Ourselves: Redesigning the Future of Humanity - One Gene at a Time. Revised paperback edition. New York, New York: Current.

Garcia, S., and Trinh, C. T. 2019. Modular Design: Implementing Proven Engineering Principles in Biotechnology. Biotechnology Advances 37: 107403. 
Gayon, J. 1998. Darwinism's Struggle for Survival: Heredity and the Hypothesis of Natural Selection. Cambridge Studies in Philosophy and Biology. Cambridge, UK; New York: Cambridge University Press.

Harris, M., and Laade, W. 1968. The Rise of Anthropological Theory: A History of Theories of Culture. Wolfgang Laade Music of Man Archive. New York: Crowell.

Heylighen, F. 2010. The Self-Organization of Time and Causality: Steps Towards Understanding the Ultimate Origin. Foundations of Science 15: 345-56.

Holton, G. J. 2000. Einstein, History, and Other Passions: The Rebellion against Science at the End of the Twentieth Century. $1^{\text {st }}$ Harvard Univ. Press paperback ed. Cambridge, Mass.: Harvard Univ. Press.

Hughes-Warrington, M. 2005. Big History. Social Evolution \& History 4 (1): $7-21$.

Hume, D. 2007. An Enquiry Concerning Human Understanding. Oxford; New York: Oxford University Press.

Huneman, P. 2019. Special Issue Editor's Introduction: 'Revisiting the Modern Synthesis.' Journal of the History of Biology 52: 509-18.

Huxley, T. H. 1860. Darwin on the Origin of Species. Westminster Review 17: $541-70$.

Jablonka, E., Lamb, M. J., and Zeligowski, A. 2014. Evolution in Four Dimensions: Genetic, Epigenetic, Behavioral, and Symbolic Variation in the History of Life. Cambridge, Massachusetts: A Bradford Book. URL: https://muse.jhu.edu/chapter/1085095.

Kaku, M. 2014. The Future of the Mind: The Scientific Quest to Understand, Enhance, and Empower the Mind. First edition. New York: Doubleday. URL: https://www.amazon.com/Future-Mind-Scientific-Understand-Enha nce/dp/038553082X.

Kardashev, N. S. 1964. Transmission of Information by Extra Terrestrial Civilizations. Soviet Astronomy 8: 217-220.

Laland, K., Uller, T., Feldman, M., Sterelny, K., Müller, G. B., Moczek, A., Jablonka, E., Odling-Smee, J., Wray, G. A., Hoekstra, H. E., Futuyma, D. J., Lenski, R. E., Mackay, T. F. C., Schluter, D., and Strassmann, J. E. 2014. Does Evolutionary Theory Need a Rethink? Nature 514: 161-64.

MacArthur, R. H. and Wilson, E. O. 2001. The Theory of Island Biogeography. Princeton: Princeton University Press.

Makin, J. G., Moses, D. A., and Chang, E. F. 2020. Machine Translation of Cortical Activity to Text with an Encoder-Decoder Framework. Nature Neuroscience. URL: http://www.nature.com/articles/s41593-020-0608-8.

Mayr, E. 1991. One Long Argument: Charles Darwin and the Genesis of Modern Evolutionary Thought. Cambridge, MA: Harvard University Press. 
Mayr, E., and Provine, W. (ed.). 1998. The Evolutionary Synthesis: Perspectives on the Unification of Biology. 4. printing. Cambridge, Mass.: Harvard Univ. Press.

Mazur, S. 2015. The Paradigm Shifters Overthrowing 'The Hegemony of the Culture of Darwin'. New York, N.Y: Caswell Books.

Mazur, S. 2019. Darwin Overthrown: Hello Mechanobiology. Caswell Books.

Morgan, L. H. 1877. Ancient Society: Researches in the Lines of Human Progress from Savagery, through Barbarism to Civilization. New York: Henry Holt and Company. URL: http://www.gutenberg.org/files/45950/4595 0-h/45950-h.htm.

National Academy of Sciences. 2020. Evolution Resources at the National Academies: Definitions of Evolutionary Terms. National Academy of Sciences. URL: https://www.nationalacademies.org/evolution/definitions.

Nazaretyan, A. P. 2005. Big (Universal) History Paradigm: Versions and Approaches Social Evolution \& History 4 (1): 61-86.

Noble, D. 2006. The Music of Life: Biology beyond the Genome. Oxford; New York: Oxford University Press.

Penrose, R. 1999. The Emperor's New Mind Concerning Computers, Minds, and the Laws of Physics. Oxford: Oxford Univ. Press.

Pigliucci, M., Müller, G., and Konrad Lorenz Institute for Evolution and Cognition Research (ed.). 2010. Evolution, the Extended Synthesis. Cambridge, Mass: MIT Press.

Pookottil, R. 2013. B.E.E.M.: Biological Emergence-based Evolutionary Mechanism: How Species Direct Their Own Evolution.

Rai, P., Prasad, A., Reddy, S. M., and Chinmay, A. 2020. Evolution of Optical Storage in Computer Memory. In Borah, S., Emilia Balas, V., and Polkowski, Z. (ed.), Advances in Data Science and Management 37 (pp. 489-95). Lecture Notes on Data Engineering and Communications Technologies. Singapore: Springer Singapore. URL: http://link.springer.com/ 10.1007/978-981-15-0978-0_48.

Richerson, P. J., and Boyd, R. 2005. Not by Genes Alone: How Culture Transformed Human Evolution. Chicago: University of Chicago Press.

Ryan, F. 2002. Darwin's Blind Spot: Evolution beyond Natural Selection. Boston: Houghton Mifflin Company.

Sahlins, M., and Service, E. (ed.). 1960. Evolution and Culture. Ann Arbor, MI: University of Michigan Press. URL: http://www.press.umich.edu/8980.

Sanders, W. 1962. Cultural Ecology of Nuclear Mesoamerica American Anthropologist 64: 34-44.

Sanderson, S. K. 1999. Social Transformations: A General Theory of Historical Development. Expanded ed. Lanham: Rowman \& Littlefield Publishers. 
Sanderson, S. K. 2007. Evolutionism and its Critics: Deconstructing and Reconstructing an Evolutionary Interpretation of Human Society. Boulder, Colo.: Paradigm Publ.

Shapiro, J. A. 2013. Evolution: A View from the 21st century. $1^{\text {st }}$ edition with corr. Upper Saddle River, NJ: FT Press Science.

Snooks, G. D. 2003. The Collapse of Darwinism, or, The Rise of a Realist Theory of Life. Lanham, Md: Lexington Books.

Snooks, G. D. 2005a. How Big History Works: Energy Flows and the Rise and Demise of Complexity. Social Evolution \& History 4 (1): 87-135.

Snooks, G. D. 2005b. The Origin of Life on Earth: A New General Dynamic Theory. Advances in Space Research 36: 226-34.

Snooks, G. D. 2007. Self-organisation or Self-creation? From Social Physics to Realist Dynamics. Social Evolution \& History 6 (1): 118-44.

Spencer, C. S. 1990. On the Tempo and Mode of State Formation: Neoevolutionism Reconsidered. Journal of Anthropological Archaeology 9: 1-30.

Steffen, W., Grinevald, J., Crutzen, P., and McNeill, J. 2011. The Anthropocene: Conceptual and Historical Perspectives. Philosophical Transactions of the Royal Society A: Mathematical, Physical and Engineering Sciences 369: 842-67.

Steward, J. H. 2008. Theory of Culture Change: The Methodology of Multilinear Evolution. Digitally reprinted from the 7. paperback pr. Urbana: Univ. of Illinois Press.

Stiegler, B. 2018a. The Neganthropocene. London: Open Humanities Press. URL: http://oapen.org/download?type=document\&docid=649431.

Stiegler, B. 2018b. Artificial Stupidity and Artificial Intelligence in the Anthropocene. November 23, Institute of Ereignis, Shanghai. URL: https://www.academia.edu/37849763/Bernard_Stiegler_Artificial_Stupidi ty_and_Artificial_Intelligence_in_the_Anthropocene_2018_.

Teilhard de Chardin, P. 1955. The Antiquity and World Expansion of Human Culture. In Thomas, W. L. (ed.), Man's Role in Changing the Face of the Earth. Chicago: University of Chicago Press.

The Third Way: Evolution in the Era of Genomics and Epigenetics 2020. URL: https://www.thethirdwayofevolution.com/. Accessed March 22.

Tizno, O., Marshall, A. R. J., Fernández-Delgado, N., Herrera, M., Molina, S. I., and Hayne, M. 2019. Room-Temperature Operation of Low-voltage, Nonvolatile, Compound-Semiconductor Memory Cells. Scientific Reports 9: 8950.

Tylor, E. B. 2010. Primitive Culture: Researches into the Development of Mythology, Philosophy, Religion, Art, and Custom. Cambridge: Cambridge University Press. URL: http://ebooks.cambridge.org/ref/id/CBO97 80511705960. 
Vernadsky, V. 1944. Problems of Biogeochemistry. Ed. by E. G. Hutchinson, trans. by G. Vernadsky. Trans Connect AcadArts Sd 35: 483-517.

Vernadsky, V. 1945. The Biosphere and the Noösphere. American Scientist 33: $1-12$.

Vernadsky, V. 2012. The Transition from the Biosphere to the Noösphere: Excerpts from Scientific Thought as a Planetary Phenomenon. Trans. by W. Jones. 21st Century, Spring-Summer: 10-31.

Whewell, W. 1840. The Philosophy of the Inductive Sciences: Founded Upon Their History. 2 vols. Volume 1. London: J.W. Parker. URL: https://books.google.co.il/books?id=Fe8TAAAAQAAJ\&printsec=frontcov er\&source $=$ gbs_ge_summary_r\&cad $=0 \# \mathrm{v}=$ onepage $\& \mathrm{q} \& \mathrm{f}=$ false.

White, L. 1943. Energy and the Evolution of Culture American Anthropologist 45: 335-56.

White, L. A. 1959. The Evolution of Culture: The Development of Civilization to the Fall of Rome. New York: McCraw-Hill Book Company, INC. URL: https://babel.hathitrust.org/cgi/pt?id=uc1.32106005438095\&view= 1 up\&seq=11.

Wilson, D. S., Hayes, S. C., Biglan, A, and Embry, D. D. 2014. Evolving the future: Toward a Science of Intentional Change. Behavioral and Brain Sciences 37: 395-416.

Wilson, E. O. 1998a. Consilience: The Unity of Knowledge. $1^{\text {st }}$ ed. New York: Knopf: Distributed by Random House.

Wilson, E. O. 1998b. Consilience among the Great Branches of Learning Daedalus 127: 131-49.

Wilson, E. O. 1999. Consilience: The Unity of Knowledge. $1^{\text {st }}$ ed. New York: Vintage Books.

Wilson, E. O. 2000. Sociobiology: The New Synthesis. $25^{\text {th }}$ anniversary ed. Cambridge, Mass: Belknap Press of Harvard University Press.

Wilson, E. O. 2004. On Human Nature. $25^{\text {th }}$ anniversary ed. Cambridge, Mass: Harvard University Press.

Wilson, E. O. 2005. Introductory Essay: Systematics and the Future of Biology. In Hey, J., Fitch, W. M., and Ayala, F. J. (ed.), National Academy of Sciences 2005. Systematics and the Origin of Species: On Ernst Mayr's $100^{\text {th }}$ Anniversary. Washington, D.C.: The National Academies Press.

Woolfson, A. 2000. Life without Genes. London: Harper Collins. 